\title{
Adaptive Finite-Time Control for a Flexible Hypersonic Vehicle with Actuator Fault
}

\author{
Jie Wang, ${ }^{1}$ Qun Zong, ${ }^{1}$ Xiao He, ${ }^{2}$ and Hamid Reza Karimi ${ }^{3}$ \\ ${ }^{1}$ Department of Electrical Engineering and Automation, Tianjin University, Tianjin 300072, China \\ ${ }^{2}$ Department of Automation, Tsinghua University, Beijing 100084, China \\ ${ }^{3}$ Department of Engineering, Faculty of Engineering and Science, University of Agder, N-4898 Grimstad, Norway
}

Correspondence should be addressed to Xiao He; hexiao@tsinghua.edu.cn

Received 6 September 2013; Accepted 3 October 2013

Academic Editor: Hongli Dong

Copyright (c) 2013 Jie Wang et al. This is an open access article distributed under the Creative Commons Attribution License, which permits unrestricted use, distribution, and reproduction in any medium, provided the original work is properly cited.

\begin{abstract}
The problem of robust fault-tolerant tracking control is investigated. Simulation on the longitudinal model of a flexible air-breathing hypersonic vehicle (FAHV) with actuator faults and uncertainties is conducted. In order to guarantee that the velocity and altitude track their desired commands in finite time with the partial loss of actuator effectiveness, an adaptive fault-tolerant control strategy is presented based on practical finite-time sliding mode method. The adaptive update laws are used to estimate the upper bound of uncertainties and the minimum value of actuator efficiency factor. Finally, simulation results show that the proposed control strategy is effective in rejecting uncertainties even in the presence of actuator faults.
\end{abstract}

\section{Introduction}

Air-breathing hypersonic vehicles (AHVs) are intended to be a reliable and cost-effective technology for access to space. Because the slender geometries and light structures cause significant flexible effects and strong coupling between propulsive and aerodynamic forces resulting from the integration of the scramjet engine, AHVs are confronting many complex problems and challenges, involving many different research areas, such as aerodynamics, thermal protection, and communication, and many problems of these fields have been reported [1-3]. Meanwhile, flight control design for AHVs is a hot topic and a challenging task $[4,5]$.

During the last decades, a kind of flexible hypersonic vehicle model including flexible dynamics has been developed in $[6,7]$. Based on this model, there have been several papers discussing the challenges associated with the control of air-breathing hypersonic vehicle (AHV) [8,9] and many control methods have been employed in the flight control system. In [10], a linear quadratic regulator (LQR) was presented for a linearized FAHV model. In [11-13], sequential loop closure controller was designed for the FAHV based on adaptive dynamic inversion together with backstepping structure. In $[14,15]$, approximate feedback linearization based on dynamic inversion method was adopted to design controller for the FAHV. In $[16,17]$, a nonlinear tracking controller was constructed by using a minimax LQR control approach, which provides robust stability and excellent tracking performance with parameter uncertainties.

The approaches mentioned above do not specifically consider possible actuator faults, which deteriorate the control performance, affect stability, and security of the AHVs, and sometimes even lead to catastrophic accidents. Consequently, it is essential that the actuator faults must be taken into account in the controller design. In the current papers, some fault-tolerant control schemes for AHVs have attracted more and more research attention and gained fruitful results, which can be reported in [18-21]. In [1820 ], the results mainly concentrate on the reentry attitude control of the AHV. Meanwhile, the fault tolerant control strategies for the longitudinal model of the AHVs are studied. In [21], an observer-based fault-tolerant control approach using both robust control and LMI techniques is designed for a linearized longitudinal AHV model in the presence of parameter uncertainties and actuator faults, but this method was effective only in the neighborhood of the operating point. On the other hand, nonlinear fault-tolerant control design methods have been devoted to the longitudinal AHV 
model. A finite-time integral sliding mode control method was proposed in [22], which could achieve superior velocity and altitude tracking performance with actuator fault. In [23], the longitudinal AHV model with unknown parameters and uncertain actuator faults is formatted into a parametric strictfeedback form, and then an adaptive fault-tolerant control scheme based on a combination of back-stepping control and dynamic surface control techniques is applied to make the velocity and altitude track the desired value.

However, the aforesaid methods only consider the rigid body of AHVs without flexible effects. A fault-tolerant control scheme for the FAHV was presented in [24], according to the model obtained by approximate linearization in given flight conditions. So, this scheme may not obtain good control performances when flight dynamics undergo great parameter perturbations. To the best of our knowledge, although considerable effort has been made on the control design for the AHVs, the important issue of fault-tolerant control of the FAHV dynamical system has not been fully investigated yet, which remains challenging and motivates us to do this study.

As a typical robust control method, sliding mode control (SMC) scheme is regarded as an effective method to cope with external disturbances and parametric uncertainties [25]. Recently, the SMC method has been widely applied for the fault tolerant control of aircraft system, spacecraft, and so on. In [26], a fault-tolerant sliding mode controller was presented for an aircraft system, which requires the message of the effectiveness factor, while it may be difficult and expensive to obtain the actuator faults online. In [27], a finite-time convergent SMC scheme is developed to solve the problem of fault-tolerant control for a rigid spacecraft. The drawback of this method is that the message of the lower bound of the effectiveness factor and the upper bound of system uncertainties needs to be known in prior.

The aforementioned references could achieve desired performance through the SMC methodology affected by actuator faults. Although the traditional SMC can guarantee the stability of the system, it adopts a linear switching function. Then the system states and the errors converge to an equilibrium point asymptotically in infinite time. In other words, it means that finite-time convergence is not ensured. Motivated by the above discussions, we propose a novel adaptive sliding mode control scheme for the longitudinal model of the FAHV with uncertainties and actuator faults in this paper. As compared with the existing results, the main contributions are as follows. Firstly, the design method of sliding mode surface based on homogeneous geometry could assure practical finite-time converged tracking of the desired command. Secondly, the upper bounds of aerodynamic uncertainties and the minimum value of actuator efficiency factor are not required in prior. The adaptive law is designed to adjust the control gains dynamically so as to ensure the establishment of sliding mode motion, and the robustness against uncertainties is ensured at the same time. After the uncertainties and actuator faults are compensated using adaptive sliding mode control scheme, the stability of the closed-loop system can be maintained.
The rest of this paper is organized as follows. In Section 2 the FAHV model is introduced and control objective is stated. Section 3 designs the sliding mode surface and the corresponding adaptive finite-time fault tolerant controller was proposed with actuator fault. Simulation results are discussed in Section 4 and the conclusions are provided in Section 5.

\section{Problem Statement}

The considered FAHV model is derived from $[6,28]$, and the longitudinal equations of motion of the FAHV are given by

$$
\begin{gathered}
\dot{V}=\frac{(T \cos \alpha-D)}{m}-g \sin \gamma, \\
\dot{\gamma}=\frac{(L+T \sin \alpha)}{m V}-\frac{g \cos \gamma}{V}, \\
\dot{h}=V \sin \gamma, \\
\dot{\alpha}=Q-\dot{\gamma}, \\
\dot{Q}=\frac{M_{y y}}{I_{y y}}, \\
\ddot{\eta}_{i}=-2 \varsigma_{m} \omega_{m, i} \dot{\eta}_{i}-\omega_{m, i}^{2} \eta_{i}+N_{i}, \quad i=1,2,3,
\end{gathered}
$$

where $x=[V, \gamma, h, \alpha, Q]^{T}$ is a vector of rigid-body state, which includes the vehicle speed, flight path angle, altitude, angel of attack, and pitch rate, respectively; $\eta_{i}, \omega_{m, i}$, and $\varsigma_{m}$ are the generalized flexible coordinate, natural frequencies, and damping coefficients of the $i$ th elastic mode. The readers may refer to [7] for a full description of the variables in this model.

Because of coupling in aerodynamic forces of the FAHV model (1), some simplifications must be carried out for the purpose of feedback linearization. The simplification of the model is necessary because we want to obtain a linearized model, and the same simplified process can be found in [29]. An input-output linearization model is developed by repeated differentiation of the outputs $V$ and $h$ as follows:

$$
\begin{aligned}
& \dddot{V}=f_{V}+b_{11} \phi_{c}+b_{12} \delta_{e}, \\
& h^{(4)}=f_{h}+b_{21} \phi_{c}+b_{22} \delta_{e},
\end{aligned}
$$

where $\phi_{c}$ and $\delta_{e}$ are control inputs and the specific expressions of $f_{V}, f_{h}, b_{11}, b_{12}, b_{21}$, and $b_{22}$ are presented in [29, equation (17)].

Compared with [29], the main propose of this study is discussing the fault tolerant controller design for the FAHV to follow a given desired output reference signals $y_{d}=\left[V_{d}, h_{d}\right]^{T}$ in the presence of partial loss of actuator effectiveness.

\section{Adaptive Finite-Time Fault-Tolerant Controller Design}

The specific controller design step includes two parts: sliding mode surface design and sliding mode control design, which can be described as follows. 
3.1. Sliding Mode Surface Design. Define tracking error variable as follows:

$$
\begin{gathered}
e_{V}=V-V_{d}, \\
e_{h}=h-h_{d} .
\end{gathered}
$$

Differentiating (4) and (5) three times, and four times respectively, results in

$$
\begin{gathered}
\dddot{e}_{V}=f_{V}-\dddot{V}_{d}+b_{11} \phi_{c}+b_{12} \delta_{e}, \\
e_{h}^{(4)}=f_{h}-h_{d}^{(4)}+b_{21} \phi_{c}+b_{22} \delta_{e} .
\end{gathered}
$$

Equations (6)-(7) can be expressed in matrix form:

$$
\left[\begin{array}{c}
\dddot{e}_{V} \\
e_{h}^{(4)}
\end{array}\right]=\underbrace{\left[\begin{array}{c}
f_{V}-\dddot{V}_{d} \\
f_{h}-h_{d}^{(4)}
\end{array}\right]}_{F=\left[F_{1}, F_{2}\right]^{T}}+\underbrace{\left[\begin{array}{ll}
b_{11} & b_{12} \\
b_{21} & b_{22}
\end{array}\right]}_{B} \underbrace{\left[\begin{array}{l}
\phi_{c} \\
\delta_{e}
\end{array}\right]}_{u}+\underbrace{\left[\begin{array}{c}
\Delta F_{1} \\
\Delta F_{2}
\end{array}\right]}_{\Delta F=\left[\Delta F_{1}, \Delta F_{2}\right]^{T}} .
$$

Note that the additional item $\Delta F$ is introduced to represent the flexible effects and coupled uncertainties described in [29, equation (14)].
Introduce new control variable:

$$
U=\left[\begin{array}{l}
U_{1} \\
U_{2}
\end{array}\right]=\left[\begin{array}{ll}
b_{11} & b_{12} \\
b_{21} & b_{22}
\end{array}\right]\left[\begin{array}{l}
\phi_{c} \\
\delta_{e}
\end{array}\right]
$$

Then (8)-(9) can be rewritten as

$$
\left[\begin{array}{c}
\dddot{e}_{V} \\
e_{h}^{(4)}
\end{array}\right]=\left[\begin{array}{l}
F_{1} \\
F_{2}
\end{array}\right]+\left[\begin{array}{l}
U_{1} \\
U_{2}
\end{array}\right]+\left[\begin{array}{l}
\Delta F_{1} \\
\Delta F_{2}
\end{array}\right]
$$

Assumption 1. The uncertainties discussed in the research are bounded $\|\Delta F\| \leq v$, but the value $v$ is unknown in advance.

Assumption 2. The matrix $B$ denoted in (8) is nonsingular over the entire flight envelope given in [12], so Assumption 1 is reasonable to be assumed.

Now, according to the definition of HOSM [30, 31], our objective is to design controller which makes the $e_{V}, e_{h}$ and their derivatives converge to the neighborhood of origin.

Design sliding mode surface as follows:

$$
\begin{gathered}
s_{V}=\ddot{e}_{V}+\int_{0}^{t} \underbrace{\lambda_{1 V}\left|e_{V}\right|^{a_{1 V}} \operatorname{sign}\left(e_{V}\right)+\lambda_{2 V}\left|\dot{e}_{V}\right|^{a_{2 V}} \operatorname{sign}\left(\dot{e}_{V}\right)+\lambda_{3 V}\left|\ddot{e}_{V}\right|^{a_{3 V}} \operatorname{sign}\left(\ddot{e}_{V}\right)}_{G_{V}}, \\
s_{h}=\dddot{e}_{h}+\int_{0}^{t} \underbrace{\lambda_{1 h}\left|e_{h}\right|^{a_{1 h}} \operatorname{sign}\left(e_{h}\right)+\lambda_{2 h}\left|\dot{e}_{h}\right|^{a_{2 h}} \operatorname{sign}\left(\dot{e}_{h}\right)+\lambda_{3 h}\left|\ddot{e}_{h}\right|^{a_{3 h}} \operatorname{sign}\left(\ddot{e}_{h}\right)+\lambda_{4 h}\left|\ddot{e}_{h}\right|^{a_{4 h}} \operatorname{sign}\left(\ddot{e}_{h}\right)}_{G_{h}} d s .
\end{gathered}
$$

The parameters $\lambda_{i V}(i=1,2,3)$ and $\lambda_{j h}(j=1,2,3,4)$ are some positive constants such that $\lambda_{3 V} s^{2}+\lambda_{2 V} s+\lambda_{1 V}$ and $\lambda_{4 h} s^{3}+\lambda_{3 h} s^{2}+\lambda_{2 h} s+\lambda_{1 h}$ are Hurwitz polynomial. The parameters $a_{i V}(i=1,2,3)$ and $a_{j h}(j=1,2,3,4)$ are determined by

$$
\begin{aligned}
& a_{(i-1) V}=\frac{a_{i V} a_{(i+1) V}}{2 a_{(i+1) V}-a_{i V}}, \quad i \in\{2,3\}, \\
& a_{(j-1) h}=\frac{a_{j h} a_{(j+1) h}}{2 a_{(j+1) h}-a_{j h}}, \quad j \in\{2,3,4\}
\end{aligned}
$$

with $a_{4 V}=a_{5 h}=1, a_{3 V} \in\left(1-\varepsilon_{V}, 1\right)$, and $a_{4 h} \in\left(1-\varepsilon_{h}, 1\right)$, where $\varepsilon_{V} \in(0,1), \varepsilon_{h} \in(0,1)$.

Based on the homogeneity theory provided in [32], it is easily shown that $e_{V}, \dot{e}_{V}, \ddot{e}_{V}$ and $e_{h}, \dot{e}_{h}, \ddot{e}_{h}, \ddot{e}_{h}$ will converge to the neighborhood of origin in finite time if it is satisfied that $s_{V}, s_{h}$ converge to the neighborhood of origin in finite time.

3.2. Adaptive Sliding Mode Controller Design. Now, let us consider the situation in which the actuator experiences partial loss of effectiveness fault. Then, differentiating (11) and (12), we obtain

$$
\left[\begin{array}{c}
\dot{s}_{V} \\
\dot{s}_{h}
\end{array}\right]=\left[\begin{array}{l}
F_{1} \\
F_{2}
\end{array}\right]+\underbrace{\left[\begin{array}{c}
G_{V} \\
G_{h}
\end{array}\right]}_{G=\left[G_{V}, G_{h}\right]^{T}}+\underbrace{\left[\begin{array}{cc}
E_{1} & 0 \\
0 & E_{2}
\end{array}\right]}_{E}\left[\begin{array}{l}
U_{1} \\
U_{2}
\end{array}\right]+\left[\begin{array}{l}
\Delta F_{1} \\
\Delta F_{2}
\end{array}\right],
$$

where $E=\operatorname{diag}\left(E_{1}, E_{2}\right) \in R^{2 \times 2}$ is a matrix characterizing the health condition of the actuators with $0 \leq E_{i} \leq 1(i=1,2)$. Note that the case $E_{i}=1$ means that the $i$ th actuator is totally healthy, the case $E_{i}=0$ implies that the $i$ th actuator completely fails, and the case $0<E_{i}<1$ corresponds to the case in which the $i$ th actuator partially loses its effectiveness, but it still has effect all the time. In this sense, the matrix $E$ becomes uncertain and even time varying but remains positive definite. In this study, an assumption $0<E_{i} \leq 1$ is given.

The control objective is to design the control inputs for $\phi_{c}$ and $\delta_{e}$ such that all of the closed-loop signals are bounded and the velocity $V$ and altitude $h$ track desired command trajectories $V_{d}$ and $h_{d}$ in the presence of flexible uncertainties and loss of effective actuator faults. That is to say, the velocity sliding mode surface $s_{V}$ and altitude sliding mode surface $s_{h}$ converge to an arbitrary small set containing the origin 
in finite time $T_{0}$, which is $\left\|s_{V}\right\| \leq \delta_{V}$ and $\left\|s_{h}\right\| \leq \delta_{h}$ for $t \geq T_{0}$, where $\delta_{V}$ and $\delta_{h}$ are arbitrary small positive constant numbers.

Let $E_{\min }=\min _{i=1,2} E_{i}$ and denote $\mu=1-E_{\min }$ and then $\mu<1$. Selecting $\theta=1 /(1-\mu)$, then the main result of the paper is formulated in the following theorem.

Theorem 3. Consider the nonlinear sliding mode dynamic system (14) with Assumptions 1 and 2, if the control $U=$ $\left[U_{1}, U_{2}\right]^{T}$ is designed as

$$
U=-F-G-k \cdot \operatorname{sig}^{\tau}(s)-\widehat{\sigma} \frac{s}{\|s\|}-\widehat{v} \frac{s}{\|s\|},
$$

with the adaptive gains

$$
\begin{gathered}
\widehat{\sigma}=-\psi+\widehat{\theta} \psi, \quad \psi=\|F\|+\|G\|+\left\|k \cdot \operatorname{sig}^{\tau}(s)\right\|+\widehat{v}, \\
\dot{\hat{\theta}}=p_{0}\left(-\varepsilon_{0} \widehat{\theta}+\psi\|s\|\right), \\
\dot{\hat{v}}=p_{1}\left(-\varepsilon_{1} \widehat{v}+\|s\|\right),
\end{gathered}
$$

where $s=\left[s_{V}, s_{h}\right]^{T}, k=\left[k_{V}, k_{h}\right]^{T}$, and $0<\tau<1$, and define the function $\operatorname{sig}^{\tau}(\cdot)=\operatorname{sign}(\cdot)|\cdot|^{\tau}, p_{0}, p_{1}, \varepsilon_{0}$ and $\varepsilon_{1}$ are positive control constants, and the initial values $\widehat{\sigma}(0), \widehat{v}(0)$ are chosen as positive constants. Then, the system trajectory will converge to the neighborhood of $s_{V}=s_{h}=0$ in finite time despite of the uncertainties $\Delta F$ and actuator faults $E$.

Proof. The stability analysis of system (14) is performed via constructing the following Lyapunov function:

$$
W=\frac{1}{2}\left(s^{T} s+\frac{1-\mu}{p_{0}}(\theta-\widehat{\theta})^{2}+\frac{1}{p_{1}}(v-\widehat{v})^{2}\right),
$$

where $\widetilde{\sigma}=\sigma-\widehat{\sigma}$ and $\widetilde{v}=v-\widehat{v}$. The derivative of (19) is presented

$$
\begin{aligned}
\dot{W}= & s^{T} \dot{s}-\frac{1-\mu}{p_{0}} \tilde{\theta} \dot{\hat{\theta}}-\frac{1}{p_{1}} \widetilde{v} \dot{v} \\
= & s^{T}(F+G+E U+\Delta F)-\frac{1-\mu}{p_{0}} \tilde{\theta} \dot{\hat{\theta}}-\frac{1}{p_{1}} \widetilde{v} \dot{v} \\
= & s^{T}(F+G+U-\underbrace{(I-E)}_{\Delta E} U+\Delta F)-\frac{1-\mu}{p_{0}} \tilde{\theta} \dot{\theta}-\frac{1}{p_{1}} \widetilde{v} \dot{v} \\
= & s^{T}(-k \cdot \operatorname{sig}^{\tau}(s)-\widehat{\sigma} \frac{s}{\|s\|}-\widehat{v} \frac{s}{\|s\|}-\underbrace{(I-E)}_{\Delta E}+\Delta F) \\
& -\frac{1-\mu}{p_{0}} \tilde{\theta} \dot{\theta}-\frac{1}{p_{1}} \widetilde{v} \dot{v} \\
\leq & -k_{V}\left|s_{V}\right|^{\tau+1}-k_{h}\left|s_{h}\right|^{\tau+1}
\end{aligned}
$$

$$
\begin{aligned}
& +s^{T}\left(-\widehat{\sigma} \frac{s}{\|s\|}-\widehat{v} \frac{s}{\|s\|}-\Delta E U+\Delta F\right)-\frac{1-\mu}{p_{0}} \tilde{\theta} \dot{\hat{\theta}}-\frac{1}{p_{1}} \widetilde{v} \dot{v} \\
\leq & -k_{V}\left|s_{V}\right|^{\tau+1}-k_{h}\left|s_{h}\right|^{\tau+1}+s^{T}\left(-\widehat{\sigma} \frac{s}{\|s\|}-\Delta E U\right) \\
& -\frac{1-\mu}{p_{0}} \tilde{\theta} \dot{\theta}+s^{T}\left(\Delta F-\widehat{v} \frac{s}{\|s\|}\right)-\frac{1}{p_{1}} \widetilde{v} \dot{v} \\
\leq & -k_{V}\left|s_{V}\right|^{\tau+1}-k_{h}\left|s_{h}\right|^{\tau+1}+s^{T}\left(-\widehat{\sigma} \frac{s}{\|s\|}-\Delta E U\right) \\
& -\frac{1-\mu}{p_{0}} \tilde{\theta} \dot{\theta}+s^{T}\left(\Delta F-v \frac{s}{\|s\|}\right)+\widetilde{v}\|s\|-\frac{1}{p_{1}} \widetilde{v} \dot{v} .
\end{aligned}
$$

In view of Assumption 1 and adaptive update laws (18), inequality (20) can be rewritten as

$$
\begin{aligned}
\dot{W} \leq & -k_{V}\left|s_{V}\right|^{\tau+1}-k_{h}\left|s_{h}\right|^{\tau+1}+s^{T}\left(-\widehat{\sigma} \frac{s}{\|s\|}-\Delta E U\right) \\
& -\frac{1-\mu}{p_{0}} \tilde{\theta} \dot{\theta}+\varepsilon_{1} \widetilde{v} \widehat{v} \\
\leq & -k_{V}\left|s_{V}\right|^{\tau+1}-k_{h}\left|s_{h}\right|^{\tau+1}-\widehat{\sigma}\|s\|-s^{T} \Delta E U \\
& -\frac{1-\mu}{p_{0}} \tilde{\theta} \dot{\theta}+\varepsilon_{1} \widetilde{v} \widehat{v} \\
\leq & -k_{V}\left|s_{V}\right|^{\tau+1}-k_{h}\left|s_{h}\right|^{\tau+1}-\widehat{\sigma}\|s\| \\
& +\|\Delta E\| \cdot s^{T} \cdot\left(\|F\|+\|G\|+\left\|k \cdot \operatorname{sig}^{\tau}(s)\right\|+\widehat{\sigma}+\widehat{v}\right) \\
& -\frac{1-\mu}{p_{0}} \tilde{\theta} \dot{\theta}+\varepsilon_{1} \widetilde{v} \widehat{v} .
\end{aligned}
$$

According to (16), inequality (21) can be rewritten as

$$
\begin{aligned}
\dot{W} \leq & -k_{V}\left|s_{V}\right|^{\tau+1}-k_{h}\left|s_{h}\right|^{\tau+1}+(1-\hat{\theta}) \psi\|s\|+\mu \cdot \widehat{\theta} \cdot \psi\|s\| \\
& -\frac{1-\mu}{p_{0}} \tilde{\theta} \dot{\theta}+\varepsilon_{1} \widetilde{v} \widehat{v} \\
\leq & -k_{V}\left|s_{V}\right|^{\tau+1}-k_{h}\left|s_{h}\right|^{\tau+1}+(1-(1-\mu) \hat{\theta}) \psi\|s\| \\
& -\frac{1-\mu}{p_{0}} \tilde{\theta} \dot{\hat{\theta}}+\varepsilon_{1} \widetilde{v} \widehat{v} \\
\leq & -k_{V}\left|s_{V}\right|^{\tau+1}-k_{h}\left|s_{h}\right|^{\tau+1} \\
& +((1-\mu) \theta-(1-\mu) \widehat{\theta}) \psi\|s\|-\frac{1-\mu}{p_{0}} \tilde{\theta} \dot{\hat{\theta}}+\varepsilon_{1} \widetilde{v} \widehat{v} \\
\leq & -k_{V}\left|s_{V}\right|^{\tau+1}-k_{h}\left|s_{h}\right|^{\tau+1}+(1-\mu) \tilde{\theta} \psi\|s\| \\
& -\frac{1-\mu}{p_{0}} \tilde{\theta} \dot{\theta}+\varepsilon_{1} \widetilde{v} \widehat{v} .
\end{aligned}
$$


According to the adaptive update laws defined in (17), the inequality (22) can be rewritten as

$$
\dot{W} \leq-k\left(\left|s_{V}\right|^{\tau+1}+\left|s_{h}\right|^{\tau+1}\right)+(1-\mu) \varepsilon_{0} \tilde{\theta} \widehat{\theta}+\varepsilon_{1} \widetilde{v} \widehat{v}
$$

where $k=\min _{i=V, h} k_{i}$. In view of Lemma 3.1 in [33], inequality (23) can be written as

$$
\begin{aligned}
\dot{W} & \leq-k\left(\left|s_{V}\right|^{2}+\left|s_{h}\right|^{2}\right)^{(\tau+1) / 2}+(1-\mu) \varepsilon_{0} \tilde{\theta} \widehat{\theta}+\varepsilon_{1} \widetilde{v} \widehat{v} \\
& \leq-k\left(\frac{1}{2}\left|s_{V}\right|^{2}+\left|s_{h}\right|^{2}\right)^{(\tau+1) / 2}+(1-\mu) \varepsilon_{0} \tilde{\theta} \widehat{\theta}+\varepsilon_{1} \widetilde{v} \widehat{v}
\end{aligned}
$$

Inspired by [33] for any positive numbers $\delta_{0}>0.5$ and $\delta_{1}>$ 0.5 , inequality (24) can be rewritten as

$$
\begin{aligned}
\dot{W} \leq & -k\left(\frac{1}{2} s^{T} s\right)^{(\tau+1) / 2}-\left(\frac{(1-\mu) \varepsilon_{0}\left(2 \delta_{0}-1\right)}{2 \delta_{0}} \widetilde{\theta}^{2}\right)^{(\tau+1) / 2} \\
& -\left(\frac{\varepsilon_{1}\left(2 \delta_{1}-1\right)}{2 \delta_{1}} \widetilde{v}^{2}\right)^{(\tau+1) / 2} \\
& +\left(\frac{(1-\mu) \varepsilon_{0}\left(2 \delta_{0}-1\right)}{2 \delta_{0}} \widetilde{\theta}^{2}\right)^{(\tau+1) / 2} \\
& +\left(\frac{\varepsilon_{1}\left(2 \delta_{1}-1\right)}{2 \delta_{1}} \widetilde{v}^{2}\right)^{(\tau+1) / 2}+(1-\mu) \varepsilon_{0} \widetilde{\theta} \widehat{\theta}+\varepsilon_{1} \widetilde{v} \widehat{v}
\end{aligned}
$$

denote

$$
p_{0}=\frac{\delta_{0} k^{2 /(\tau+1)}}{\varepsilon_{0}\left(2 \delta_{0}-1\right)}, \quad p_{1}=\frac{\delta_{1} k^{2 /(\tau+1)}}{\varepsilon_{1}\left(2 \delta_{1}-1\right)} .
$$

Then, inequality (25) can be rewritten as

$$
\begin{aligned}
\dot{W} \leq & -k\left[\left(\frac{1}{2} s^{T} s\right)^{(\tau+1) / 2}+\left(\frac{(1-\mu)}{2 p_{0}} \widetilde{\theta}^{2}\right)^{(\tau+1) / 2}\right. \\
& \left.+\left(\frac{1}{2 p_{1}} \widetilde{v}^{2}\right)^{(\tau+1) / 2}\right] \\
& +\left(\frac{(1-\mu) \varepsilon_{0}\left(2 \delta_{0}-1\right)}{2 \delta_{0}} \widetilde{\theta}^{2}\right)^{(\tau+1) / 2} \\
& +\left(\frac{\varepsilon_{1}\left(2 \delta_{1}-1\right)}{2 \delta_{1}} \widetilde{v}^{2}\right)^{(\tau+1) / 2}+(1-\mu) \varepsilon_{0} \widetilde{\theta} \hat{\theta}+\varepsilon_{1} \widetilde{v} \widehat{v} .
\end{aligned}
$$

According to Lemma 3.2 in [33], when $\delta_{0}>0.5, \delta_{1}>0.5$, and $0.5<0.5(\tau+1)<1$, the time derivative of the Lyapunov function $\dot{W}$ becomes

$$
\begin{aligned}
\dot{W} \leq & -k\left[\left(\frac{1}{2} s^{T} s\right)+\left(\frac{(1-\mu)}{2 p_{0}} \widetilde{\theta}^{2}\right)+\left(\frac{1}{2 p_{1}} \widetilde{v}^{2}\right)\right]^{(\tau+1) / 2} \\
& +\left(\frac{(1-\mu) \varepsilon_{0}\left(2 \delta_{0}-1\right)}{2 \delta_{0}} \widetilde{\theta}^{2}\right)^{(\tau+1) / 2} \\
& +\left(\frac{\varepsilon_{1}\left(2 \delta_{1}-1\right)}{2 \delta_{1}} \widetilde{v}^{2}\right)^{(\tau+1) / 2} \\
& +(1-\mu) \varepsilon_{0} \widetilde{\theta} \hat{\theta}+\varepsilon_{1} \widetilde{v} \widehat{v} \\
\leq & -k W^{(\tau+1) / 2}+\left(\frac{(1-\mu) \varepsilon_{0}\left(2 \delta_{0}-1\right)}{2 \delta_{0}} \widetilde{\theta}^{2}\right)^{(\tau+1) / 2} \\
& +\left(\frac{\varepsilon_{1}\left(2 \delta_{1}-1\right)}{2 \delta_{1}} \widetilde{v}^{2}\right)^{(\tau+1) / 2}+(1-\mu) \varepsilon_{0} \tilde{\theta} \widehat{\theta}+\varepsilon_{1} \widetilde{v} \widehat{v} .
\end{aligned}
$$

Note that, for any positive constants $\delta_{0}>0.5$ and $\delta_{1}>0.5$, the following inequality holds:

$$
\begin{aligned}
\varepsilon_{1} \widetilde{v} \widehat{v} & =\varepsilon_{1}\left(-\widetilde{v}^{2}+\widetilde{v} v\right) \\
& \leq \varepsilon_{1}\left(-\widetilde{v}^{2}+\frac{1}{2 \delta_{1}} \widetilde{v}^{2}+\frac{\delta_{1}}{2} v^{2}\right) \\
& \leq \frac{-\varepsilon_{1}\left(2 \delta_{1}-1\right)}{2 \delta_{1}} \widetilde{v}^{2}+\frac{\varepsilon_{1} \delta_{1}}{2} v^{2} .
\end{aligned}
$$

Similarly $(1-\mu) \varepsilon_{0} \widetilde{\theta} \widehat{\theta}$ satisfies the following inequality:

$$
(1-\mu) \varepsilon_{0} \widetilde{\theta} \widehat{\theta} \leq \frac{-\varepsilon_{0}(1-\mu)\left(2 \delta_{0}-1\right)}{2 \delta_{0}} \widetilde{\theta}^{2}+\frac{\varepsilon_{0}(1-\mu) \delta_{0}}{2} \theta^{2} .
$$

According to inequality (29), if $\left(\varepsilon_{1}\left(2 \delta_{1}-1\right) / 2 \delta_{1}\right) \widetilde{v}^{2}>1$, we obtain

$$
\begin{aligned}
& \left(\frac{\varepsilon_{1}\left(2 \delta_{1}-1\right)}{2 \delta_{1}} \widetilde{v}^{2}\right)^{(\tau+1) / 2}+\varepsilon_{1} \widetilde{v} \widehat{v} \\
& \leq \frac{\varepsilon_{1}\left(2 \delta_{1}-1\right)}{2 \delta_{1}} \widetilde{v}^{2}+\varepsilon_{1} \widetilde{v} \hat{v} \leq \frac{\varepsilon_{1} \delta_{1}}{2} v^{2} .
\end{aligned}
$$

If $\left(\varepsilon_{1}\left(2 \delta_{1}-1\right) / 2 \delta\right) \widetilde{v}^{2} \leq 1$, we have

$$
\begin{gathered}
\left.\left(\frac{\varepsilon_{1}\left(2 \delta_{1}-1\right)}{2 \delta_{1}} \widetilde{v}^{2}\right)^{(\tau+1) / 2}\right|_{\left(\varepsilon_{1}\left(2 \delta_{1}-1\right) / 2 \delta_{1}\right) \widetilde{v}^{2} \leq 1} \\
\quad<\left.\left(\frac{\varepsilon_{1}\left(2 \delta_{1}-1\right)}{2 \delta_{1}} \widetilde{v}^{2}\right)^{(\tau+1) / 2}\right|_{\left(\varepsilon_{1}\left(2 \delta_{1}-1\right) / 2 \delta_{1}\right) \widetilde{v}^{2}>1} .
\end{gathered}
$$

Therefore, combining (31) and (32) yields

$$
\left(\frac{\varepsilon_{1}\left(2 \delta_{1}-1\right)}{2 \delta_{1}} \widetilde{v}^{2}\right)^{(\tau+1) / 2}+\varepsilon_{1} \widetilde{v} \hat{v} \leq \frac{\varepsilon_{1} \delta_{1}}{2} v^{2} .
$$



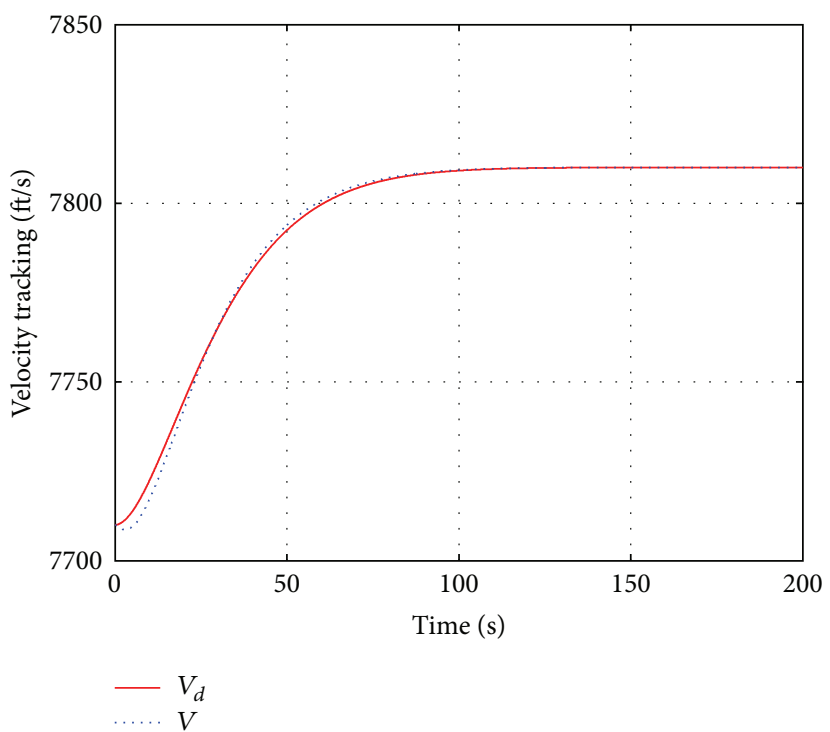

(a)

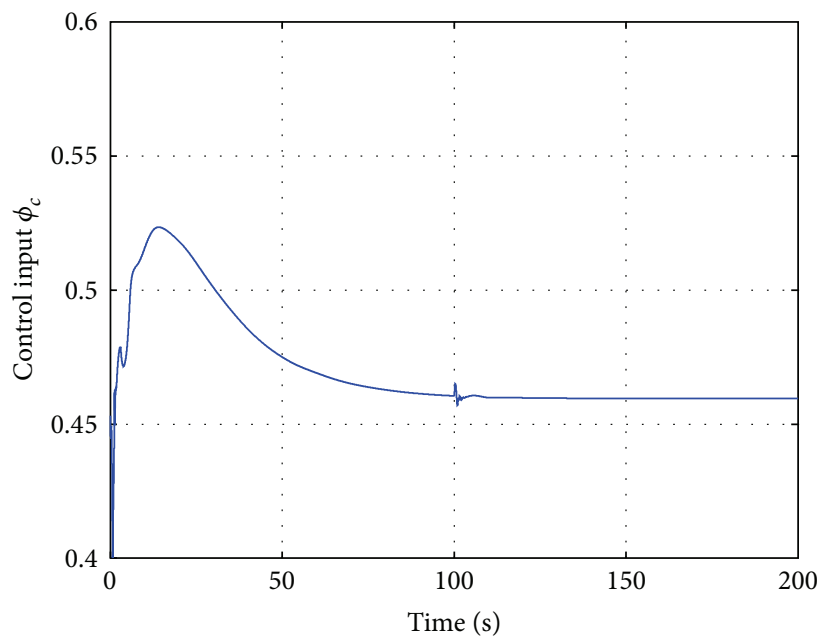

(c)

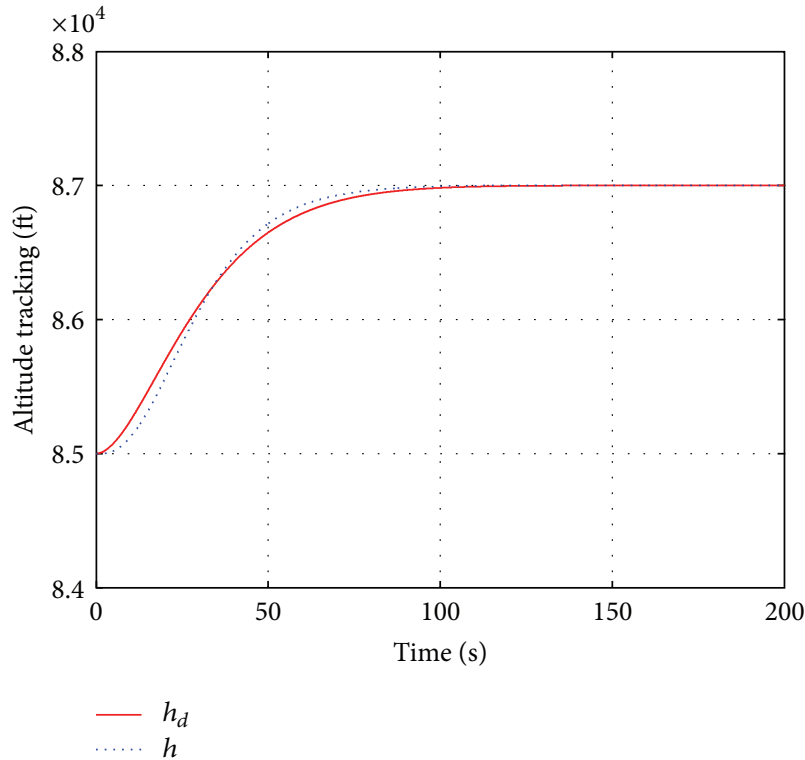

(b)

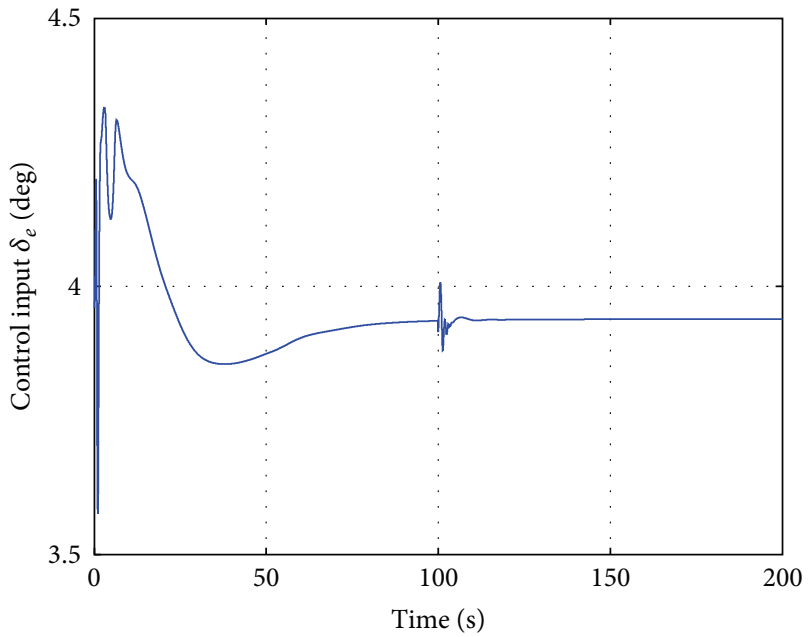

(d)

FIGURE 1: Regulated outputs and control inputs with actuator faults.

Similar to (33), the following inequality can be obtained:

$$
\begin{gathered}
\left(\frac{(1-\mu) \varepsilon_{0}\left(2 \delta_{0}-1\right)}{2 \delta_{0}} \widetilde{\theta}^{2}\right)^{(\tau+1) / 2}+(1-\mu) \varepsilon_{0} \widetilde{\theta} \widehat{\theta} \\
\leq \frac{(1-\mu) \varepsilon_{0} \delta_{0}}{2} \theta^{2} .
\end{gathered}
$$

Thus, from (28)-(34), the derivative of the Lyapunov function (28) becomes

$$
\dot{W} \leq-k W^{(\tau+1) / 2}+\mu_{0},
$$

where

$$
\mu_{0}=\frac{\varepsilon_{1} \delta_{1}}{2} v^{2}+\frac{(1-\mu) \varepsilon_{0} \delta_{0}}{2} \theta^{2}
$$

According to Lemma 3.6 in [33], the decrease of $W$ can drive the sliding mode surfaces $s_{V}$ and $s_{h}$ to converge to a neighborhood of the sliding surface in finite time. Furthermore, selecting $0<\beta \leq 1$, inequality (35) can be expressed as

$$
\dot{W} \leq-\beta k W^{(\tau+1) / 2}-(1-\beta) k W^{(\tau+1) / 2}+\mu_{0} .
$$

If $-(1-\beta) k W^{(\tau+1) / 2}+\mu_{0}<0$, then $\dot{W} \leq-\beta k W^{(\tau+1) / 2}$. Based on the conclusion from [30], the decrease of $W$ drives the trajectories of the closed-loop system into $W^{(\tau+1) / 2} \leq$ $\mu_{0} /(1-\beta) k$. Therefore, the trajectories of the closed-loop system is bounded in finite time as

$$
\lim _{\beta \rightarrow \beta_{0}} s(t) \in(\|s\| \leq \underbrace{\left(\frac{\mu_{0}}{\left(1-\beta_{0}\right) k}\right)^{1 /(\tau+1)}}_{\varepsilon}),
$$




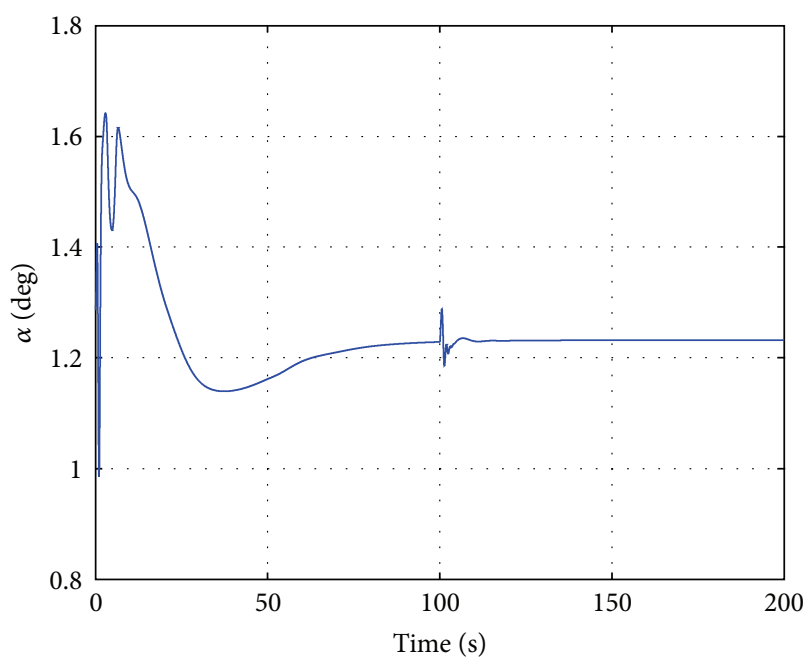

(a)

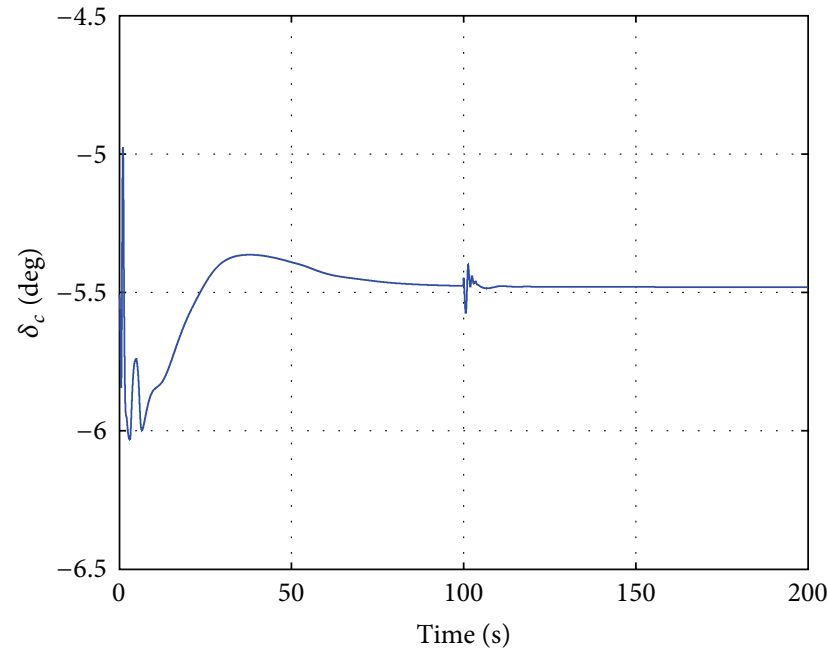

(c)

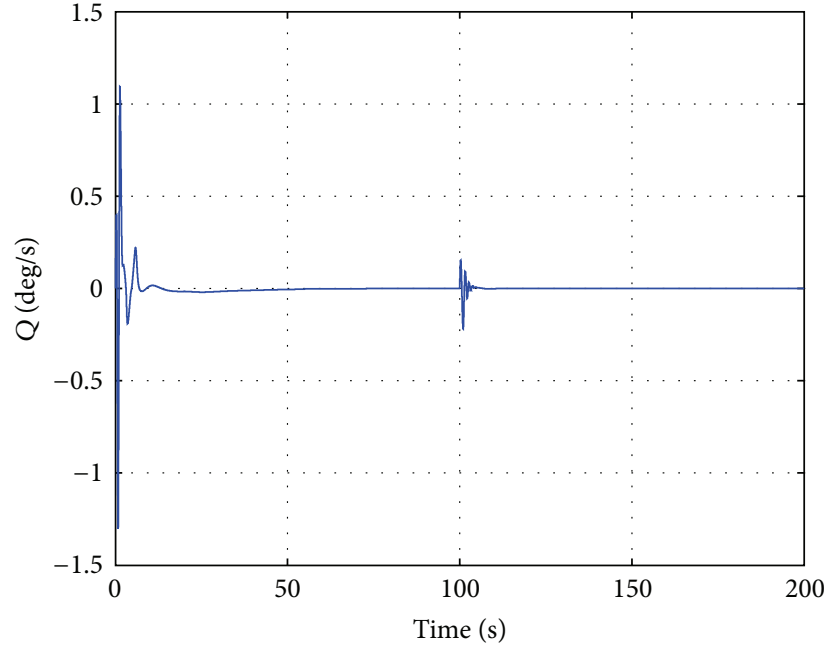

(b)

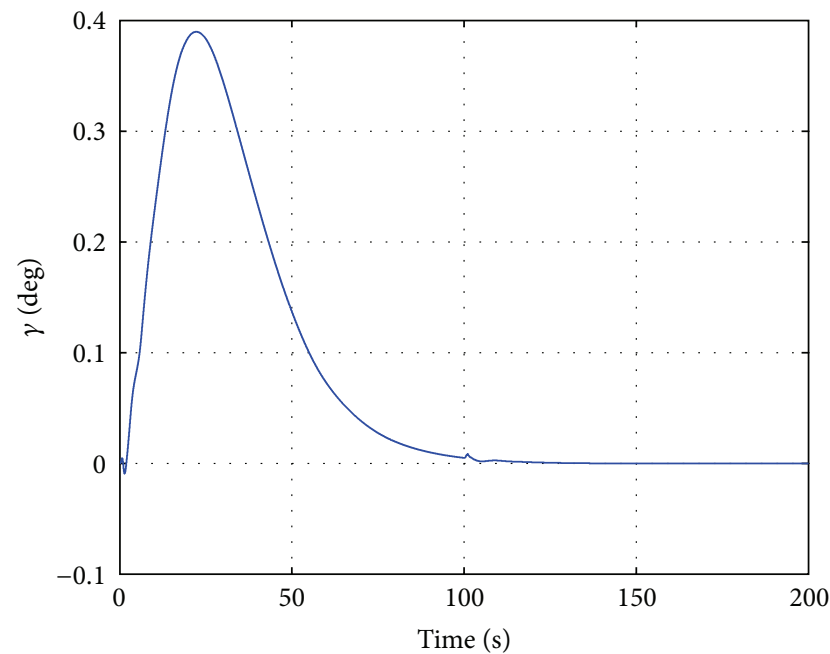

(d)

FIGURE 2: Other flight states with actuator faults.

where $0<\beta_{0}<1$ and $\varepsilon$ is a small set containing the origin of the closed-loop system. And the time needed to reach (38) is bound as

$$
T \leq \frac{2 W(0)^{(1-\tau) / 2}}{k \beta_{0}(1-\tau)},
$$

where $W(0)$ is the initial value of $W$. After that, the control objective that the $e_{V}, \dot{e}_{V}, \ddot{e}_{V}$ and $e_{h}, \dot{e}_{h}, \ddot{e}_{h}, \ddot{e}_{h}$ converge to the neighborhood of origin is established.

When the control $U=\left[U_{1}, U_{2}\right]^{T}$ is designed via (9), according to Assumption 2 the actual control variable is calculated as

$$
\left[\begin{array}{l}
\phi_{c} \\
\delta_{e}
\end{array}\right]=\left[\begin{array}{ll}
b_{11} & b_{12} \\
b_{21} & b_{22}
\end{array}\right]^{-1}\left[\begin{array}{l}
U_{1} \\
U_{2}
\end{array}\right]
$$

It is evident from (40) that the finite-time convergent performance of the proposed adaptive fault tolerant controller can be obtained without the knowledge of the minimum value of actuator effectiveness factor. Meanwhile, the upper bound of uncertainties does not need to be known in advance.

\section{Simulation}

To illustrate the efficiency of controller designed previously, a climbing maneuver with longitudinal acceleration for a $100 \mathrm{ft} / \mathrm{s}$ velocity change and a $1000 \mathrm{ft}$ altitude change is considered. Simulation studies have been done on the full nonlinear flexible hypersonic vehicle defined in (1). The reference commands have been generated by filtering step reference commands by a second-order prefilter with natural frequency $\omega_{f}=0.06 \mathrm{rad} / \mathrm{s}$ and damping ratio $\zeta_{f}=0.95$.

The initial trim condition is selected as $V=7710 \mathrm{ft} / \mathrm{s}$ and $h=85000 \mathrm{ft}$. Simulation parameters are provided in Table 1 . 


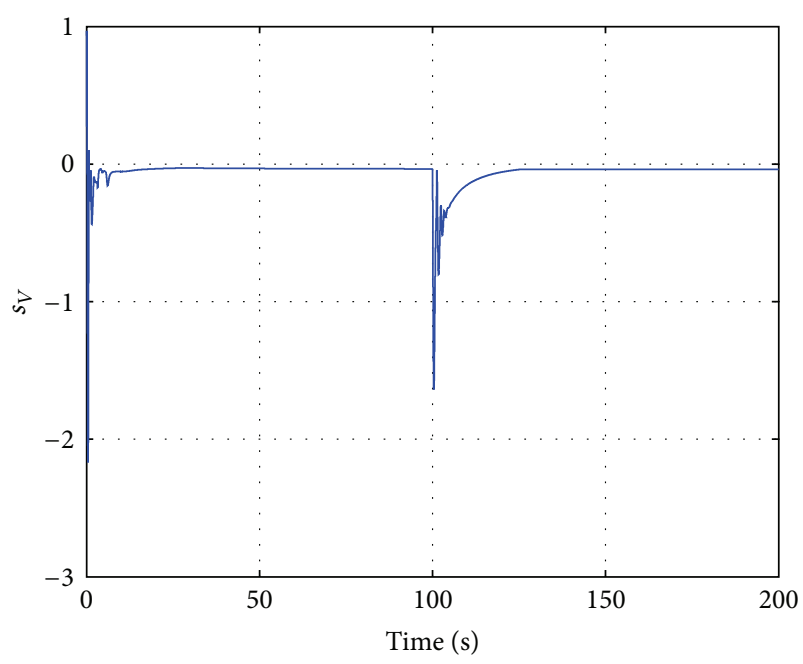

(a)

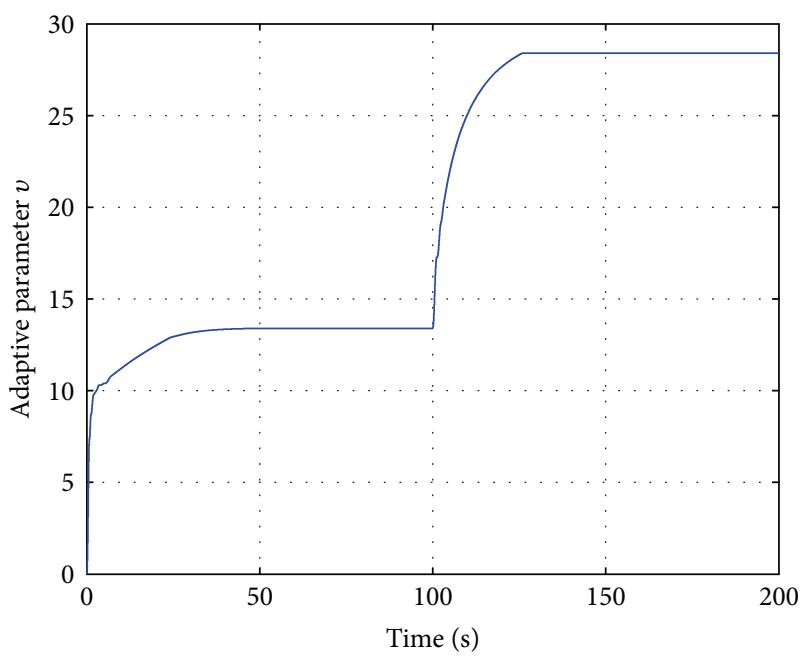

(c)

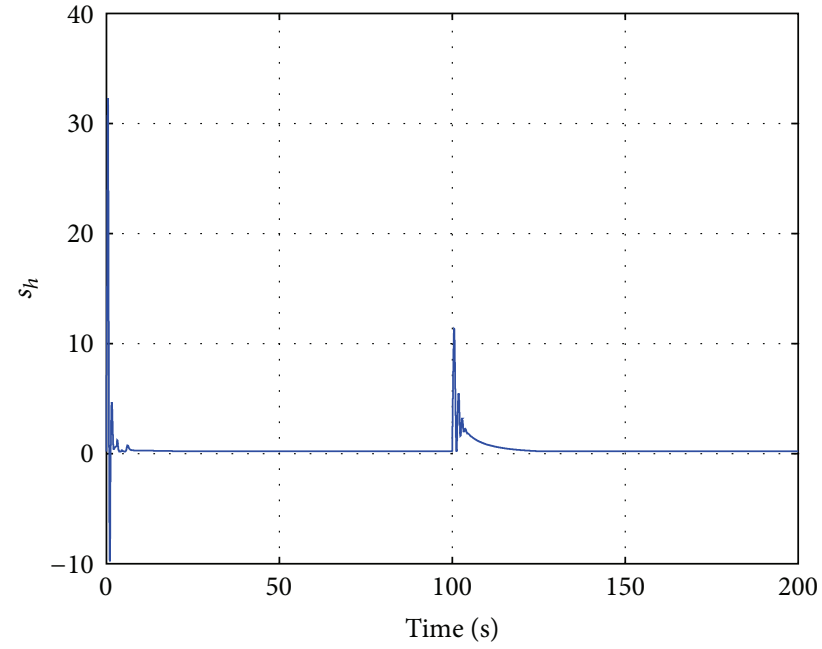

(b)

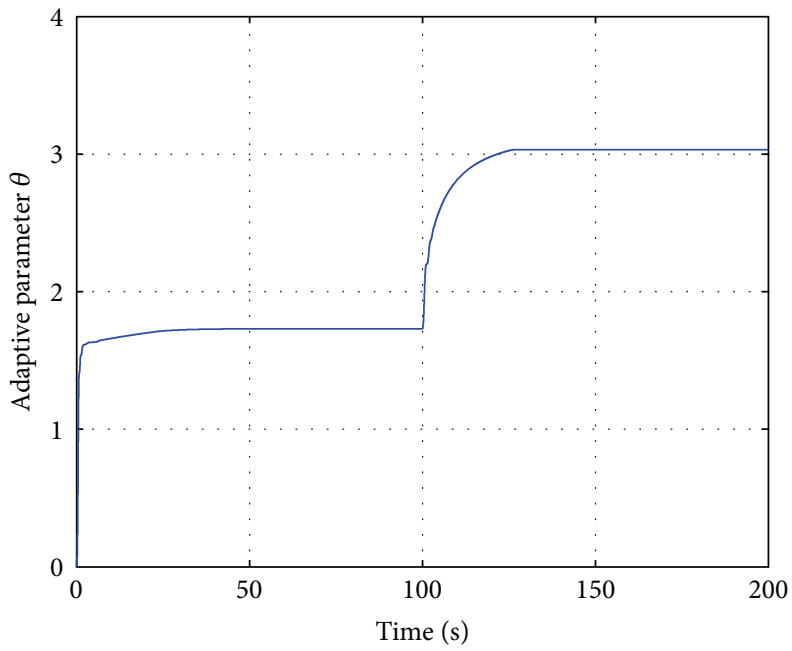

(d)

FIGURE 3: Sliding mode surface and adaptive parameters $\widehat{v}$ and $\widehat{\theta}$ with actuator faults.

TABLE 1: Simulation parameters setting.

\begin{tabular}{lccccc}
\hline Items & Values & Items & Values & Items & Values \\
\hline$\lambda_{1 V}$ & 10 & $\lambda_{4 h}$ & 10 & $a_{3 h}$ & $3 / 5$ \\
$\lambda_{2 V}$ & 15 & $a_{1 V}$ & $1 / 2$ & $a_{4 h}$ & $3 / 4$ \\
$\lambda_{3 V}$ & 15 & $a_{2 V}$ & $3 / 5$ & $\tau$ & 0.7 \\
$\lambda_{1 h}$ & 15 & $a_{3 V}$ & $3 / 4$ & $k_{V}$ & 10 \\
$\lambda_{2 h}$ & 25 & $a_{1 h}$ & $3 / 7$ & $k_{h}$ & 10 \\
$\lambda_{3 h}$ & 20 & $a_{2 h}$ & $1 / 2$ & & \\
\hline
\end{tabular}

It is assumed that actuator faults are chosen as

$$
\begin{aligned}
& E_{1}=0.7, \quad t \geq 100, \\
& E_{2}=0.7, \quad t \geq 100 .
\end{aligned}
$$

The simulation results are provided in Figures 1-4. Figure 1 denotes the response to the $100 \mathrm{ft} / \mathrm{s}$ step velocity and $1000 \mathrm{ft}$ step altitude. It has been observed that the velocity and altitude converge to the desired value. The control inputs of $\phi_{c}$ and $\delta_{e}$ could be seen in bottom plots of Figure 1 .

Figure 2 shows the performance of the angle of attack $\alpha$ and the pitch rate $Q$ at the top, as well as the canard deflection $\delta_{c}$ and the flight path angle $\gamma$ at the bottom.

The velocity and altitude sliding mode surfaces $s_{V}, s_{h}$ are shown in Figure 3, which are oscillation with small magnitudes when actuator fault occurred. The convergent performance verifies the effectiveness of the proposed control strategy. The adaptive parameters $\widehat{v}$ and $\widehat{\theta}$ in control laws of (15)-(18) could be seen in bottom plots of Figure 3, where the convergence of $\widehat{v}$ is confirmed. From the simulation results in Figure 3 , the approximate equation $\widehat{\theta} \approx 3$ can be obtained. According to the relationship based on equation $\theta=1 /(1-\mu)$, we can solve that $\widehat{\mu}=0.67$ and denote the estimated error as

$$
e_{\mu}=\mu-\widehat{\mu} \approx 0.7-0.67=0.03
$$




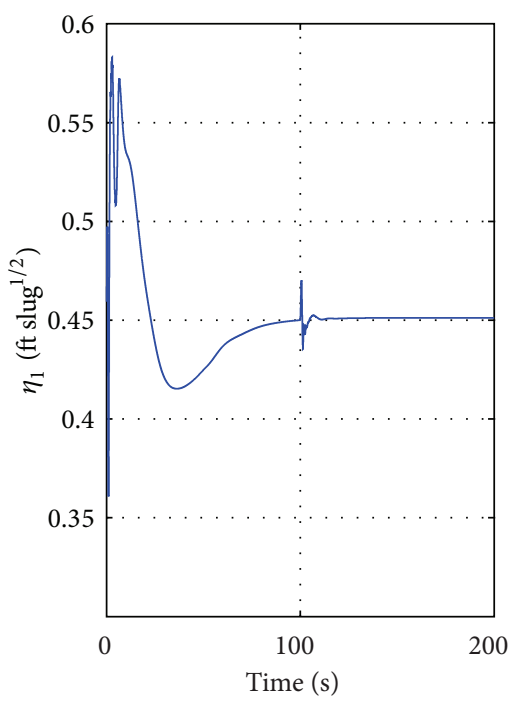

(a)

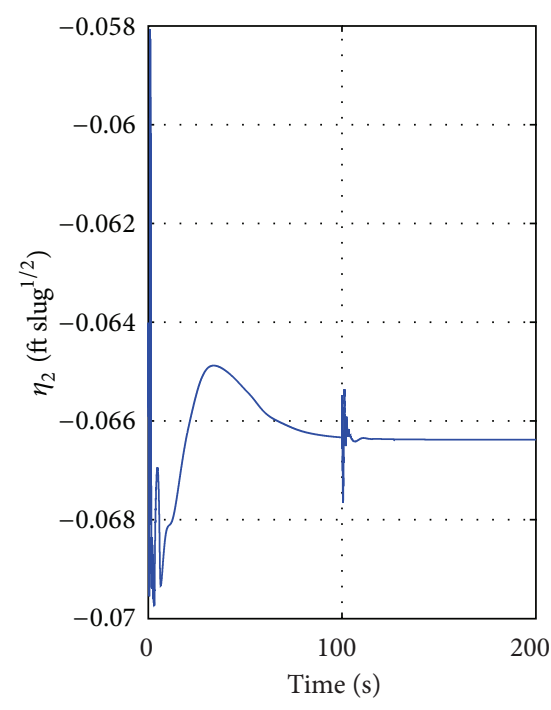

(b)

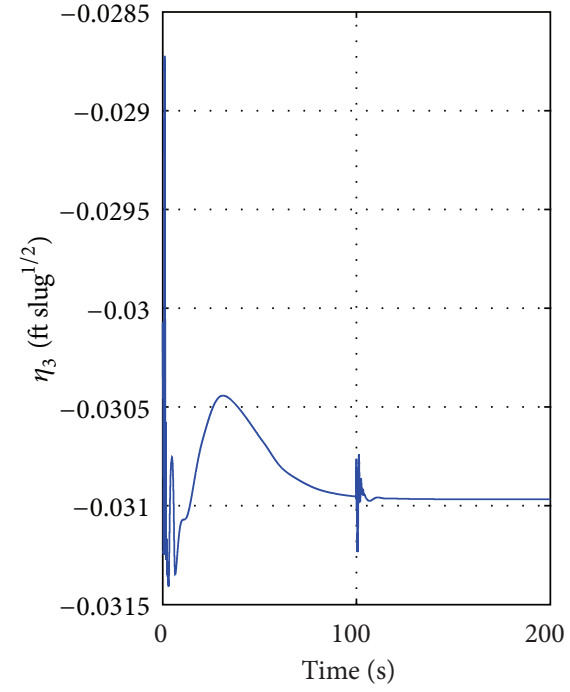

(c)

FIgURE 4: The dynamic response curves of flexile modes with actuator faults.

The value of $e_{\mu}$ in our research is in tolerance. Meanwhile, the stability of flexible states is depicted by Figure 4 . And it can be seen that the flexible states $\eta_{1}, \eta_{2}$, and $\eta_{3}$ converge to constant values, respectively.

In summary, the simulation results demonstrate that, although there are actuator faults and uncertainties in the system, the good tracking performance and satisfactory system responses can be guaranteed.

\section{Conclusions and Future Work}

In this paper, an effective method has been proposed for linearizing the nonlinear model of the FAHV via feedback, which simplifies the complexity of the controller design process. Furthermore, an adaptive fault-tolerant control scheme based on finite-time sliding mode control technique has been brought forward for the FAHV without any information about the upper bound of uncertainties or the minimum value of actuator effectiveness. Simulation results have been presented to evaluate the validity of the proposed control scheme and to show its robustness to uncertainties and the loss of actuator effectiveness.

Further research work includes two aspects. Firstly, only the loss-of-effectiveness fault has been investigated in this paper; other types of actuator faults such as float failure and actuator faults in FAHV with unknown structure are worth being dealt with. Furthermore, the FAHV model considered in this paper is highly nonlinear and strongly coupled, and a more general active FTC scheme as adaptive fault diagnosis observer in $[34,35]$ should be investigated in our future study.

\section{Conflict of Interests}

The authors declare that there is no conflict of interests regarding the publication of this paper.

\section{Acknowledgments}

This research was supported in part by National Natural Science Foundation of China (nos. 91016018, 61004073, and 61203119), the Foundation for Key Program of Ministry of Education, China (no. 311012), the Key Program for Basic Research of Tianjin (no. 11JCZDJC25100), and the Key Program of Tianjin Natural Science (no. 12JCZDJC30300), and Aeronautical Science Foundation of China (no. 20125848004) supported by Science and Technology on Aircraft Control Laboratory.

\section{References}

[1] A. Mehrsai, H. R. Karimi, and K. D. Thoben, "Integration of supply networks for customization with modularity in cloud and make-to-upgrade strategy," Systems Science and Control Engineering, vol. 1, no. 1, pp. 28-42, 2013.

[2] S. R. Desai and R. Prasad, "A new approach to order reduction using stability equation and big bang big crunch optimization," Systems Science and Control Engineering, vol. 1, no. 1, pp. 20-27, 2013.

[3] Y. Chen and K. A. Hoo, "Stability analysis for closed-loop management of a reservoir based on identification of reduced-order nonlinear model," Systems Science and Control Engineering, vol. 1, no. 1, pp. 12-19, 2013.

[4] B. Fidan, M. Mirmirani, and P. A. Ioannou, "Flight dynamics and control of air-breathing hypersonic vehicles: review and new direction," in Proceedings of the 12th AIAA International Space Planes and Hypersonic Systems and Technologies, pp. 2003-7081, Norfolk, UK, December 2003.

[5] M. A. Bolender and D. B. Doman, "Nonlinear longitudinal dynamical model of an air-breathing hypersonic vehicle," Journal of Spacecraft and Rockets, vol. 44, no. 2, pp. 374-387, 2007.

[6] T. Williams, M. A. Bolender, D. B. Doman, and O. Morataya, "An aerothermal flexible mode analysis of a hypersonic vehicle," 
in Proceesings of the Atmospheric Flight Mechanics Conference, pp. 1391-1412, August 2006.

[7] D. O. Sigthorsson and A. Serrani, "Development of linear parameter-varying models of hypersonic air-breathing vehicles," in Proceedings of the AIAA Guidance, Navigation, and Control Conference and Exhibit, Chicago, Ill, USA, August 2009.

[8] M. Kuipers, M. Mirmirani, P. Ioannou, and Y. Huo, "Adaptive control of an aeroelastic airbreathing hypersonic cruise vehicle," in Proceedings of the AIAA Guidance, Navigation, and Control Conference, pp. 235-246, Hilton Head, SC, USA, August 2007.

[9] H. B. Duan and P. Li, "Progress in control approaches for hypersonic vehicle," Science China, vol. 55, no. 10, pp. 29652970, 2012.

[10] K. P. Groves, D. O. Sigthorsson, A. Serrani, S. Yurkovich, M. A. Bolender, and D. B. Doman, "Reference command tracking for a linearized model of an air-breathing hypersonic vehicle," in Proceedings of the AIAA Guidance, Navigation, and Control Conference, pp. 2901-2914, August 2005.

[11] L. Fiorentini, A. Serrani, M. A. Bolender, and D. B. Doman, "Robust nonlinear sequential loop closure control design for an air-breathing hypersonic vehicle model," in Proceedings of the American Control Conference (ACC '08), pp. 3458-3463, Seattle, Wash, USA, June 2008.

[12] L. Fiorentini, A. Serrani, M. A. Bolender, and D. B. Doman, "Nonlinear robust adaptive control of flexible air-breathing hypersonic vehicles," Journal of Guidance, Control, and Dynamics, vol. 32, no. 2, pp. 401-416, 2009.

[13] L. Fiorentini, Nonlinear Adaptive Controller Design for Airbreathing Hypersonic Vehicles [M.S. thesis], Ohio State University, 2010.

[14] J. T. Parker, A. Serrani, S. Yurkovich, M. A. Bolender, and D. B. Doman, "Approximate feedback linearization of an airbreathing hypersonic vehicle," in Proceedings of the AIAA Guidance, Navigation, and Control Conference, pp. 3633-3648, usa, August 2006.

[15] J. T. Parker, A. Serrani, S. Yurkovich, M. A. Bolender, and D. B. Doman, "Control-oriented modeling of an air-breathing hypersonic vehicle," Journal of Guidance, Control, and Dynamics, vol. 30, no. 3, pp. 856-869, 2007.

[16] O. U. Rehman, B. Fidan, and I. Petersen, "Uncertainty modeling for robust minimax LQR control of hypersonic flight vehicles," in Proceedings of the AIAA Guidance, Navigation, and Control Conference and Exhibit, October 2009.

[17] O. U. Rehman, I. R. Petersen, and B. Fidan, "Robust nonlinear control of a nonlinear uncertain system with input coupling and its application to hypersonic flight vehicles," in Proceedings of the IEEE International Conference on Control Applications, CCA 2010, pp. 1451-1457, Yokohama, Japan, September 2010.

[18] Z. Gao, B. Jiang, R. Qi, and Y. Xu, "Robust reliable control for a near space vehicle with parametric uncertainties and actuator faults," International Journal of Systems Science, vol. 42, no. 12, pp. 2113-2124, 2011.

[19] Z. Gao, B. Jiang, P. Shi, M. Qian, and J. Lin, "Active fault tolerant control design for reusable launch vehicle using adaptive sliding mode technique," Journal of the Franklin Institute, vol. 349, no. 4, pp. 1543-1560, 2012.

[20] Z. F. Gao and B. Jiang, "Active fault tolerant control design for near-space vehicle attitude dynamic with actuator faults," Proceedings of the IMechE I, vol. 225, no. 3, pp. 413-422, 2012.

[21] Z. Gao, B. Jiang, P. Shi, J. Liu, and Y. Xu, "Passive fault-tolerant control design for near-space hypersonic vehicle dynamical system," Circuits, Systems, and Signal Processing, vol. 31, no. 2, pp. 565-581, 2012.

[22] H. Li, L. Wu, Y. Si, H. Gao, and X. Hu, "Multi-objective fault-tolerant output tracking control of a flexible air-breathing hypersonic vehicle," Proceedings of the Institution of Mechanical Engineers I, vol. 224, no. 6, pp. 647-667, 2010.

[23] R. Y. Qi, Y. H. Huang, and B. Jiang, “Adaptive back-stepping control for a hypersonic vehicle with uncertain parameters and actuator faults," Proceedings of the IMechE I, vol. 227, no. 1, pp. 51-61, 2013.

[24] S. H. Li, H. B. Sun, and C. Y. Sun, "Robust adaptive integral sliding mode fault-tolerant control for airbreathing hypersonic vehicles," Proceedings of the IMechE I, vol. 226, no. 10, pp. 13441355, 2012.

[25] V. I. Utkin, "Variable structure systems with sliding modes," IEEE Transactions on Automatic Control, vol. 22, no. 2, pp. 212$222,1977$.

[26] H. Alwi, C. Edwards, O. Stroosma, and J. A. Mulder, "Fault tolerant sliding mode control design with piloted simulator evaluation," Journal of Guidance, Control, and Dynamics, vol. 31, no. 5, pp. 1186-1201, 2008.

[27] Q. L. Hu and B. Xiao, "Robust finite-time control for spacecraft attitude stabilization under actuator fault," Proceedings of the IMechE I, vol. 226, no. 13, pp. 416-428, 2013.

[28] M. A. Bolender, "An overview on dynamics and controls modelling of hypersonic vehicles," in Proceedings of the American Control Conference (ACC '09), pp. 2507-2512, St. Louis, MO, USA, June 2009.

[29] B. Tian, W. Fan, Q. Zong, J. Wang, and F. Wang, "Adaptive high order sliding mode controller design for hypersonic vehicle with flexible body dynamics," Mathematical Problems in Engineering, vol. 2013, Article ID 357685, 11 pages, 2013.

[30] A. Levant, "Homogeneous high-order sliding modes," in Proceedings of the 17th IFAC World Congress, pp. 3799-3810, Seoul, Korea, 2008.

[31] A. Levant, "Finite-time stability and high relative degrees in sliding-mode control," in Sliding Modes After the First Decade of the 21st Century, vol. 412 of Lecture Notes in Control and Information Sciences, pp. 59-92, 2012.

[32] S. P. Bhat and D. S. Bernstein, "Geometric homogeneity with applications to finite-time stability," Mathematics of Control, Signals, and Systems, vol. 17, no. 2, pp. 101-127, 2005.

[33] Z. Zhu, Y. Xia, and M. Fu, "Attitude stabilization of rigid spacecraft with finite-time convergence," International Journal of Robust and Nonlinear Control, vol. 21, no. 6, pp. 686-702, 2011.

[34] S. Yin, S. Ding, A. Haghani, and H. Hao, "A comparison study of basic data driven fault diagnosis and process monitoring methods on the benchmark Tennessee Eastman process," Journal of Process Control, vol. 22, no. 9, pp. 1567-1581, 2012.

[35] S. Yin, H. Luo, and S. Ding, "Real-time implementation of faulttolerant control systems with performance optimization," IEEE Transactions on Industrial Electronics, vol. 61, no. 5, pp. 24022411, 2013. 


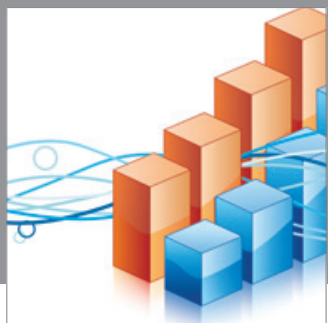

Advances in

Operations Research

mansans

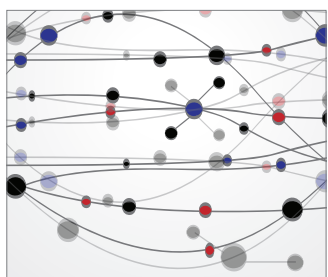

The Scientific World Journal
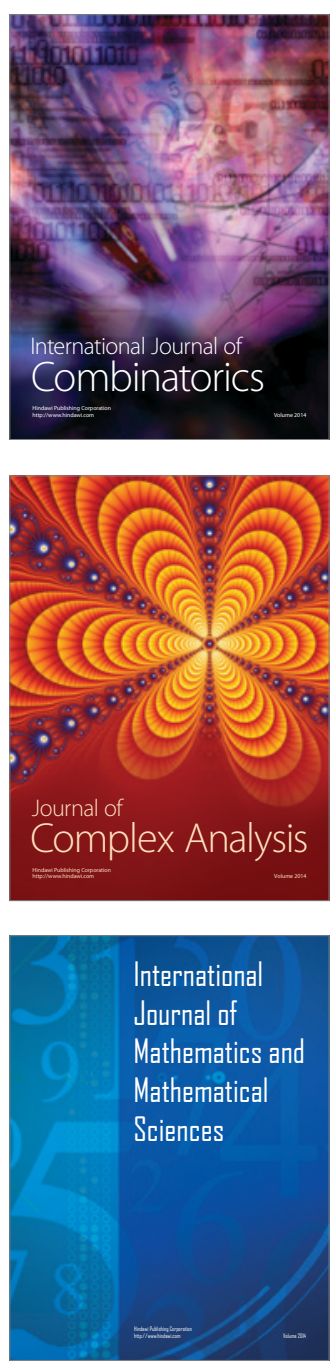
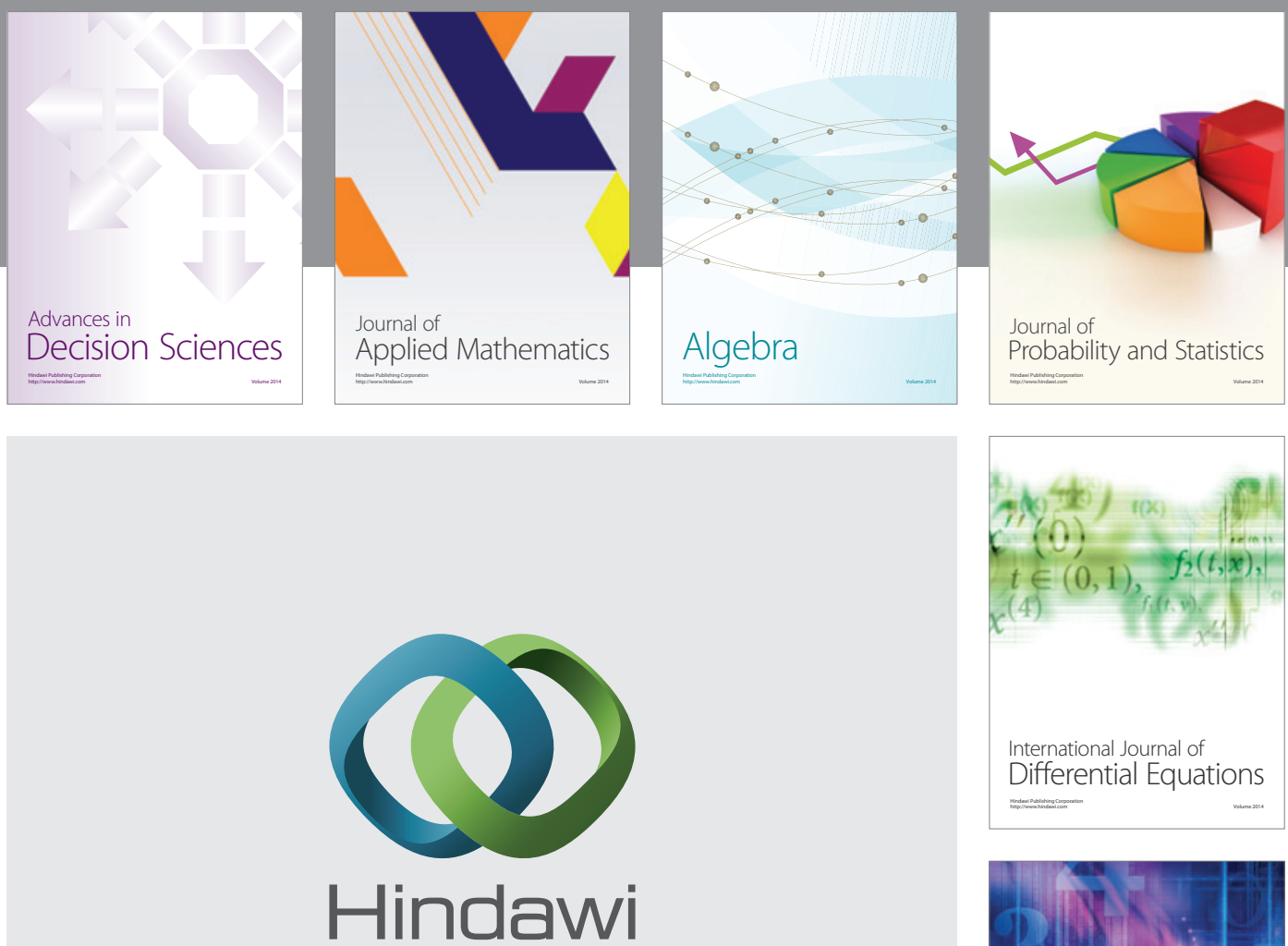

Submit your manuscripts at http://www.hindawi.com
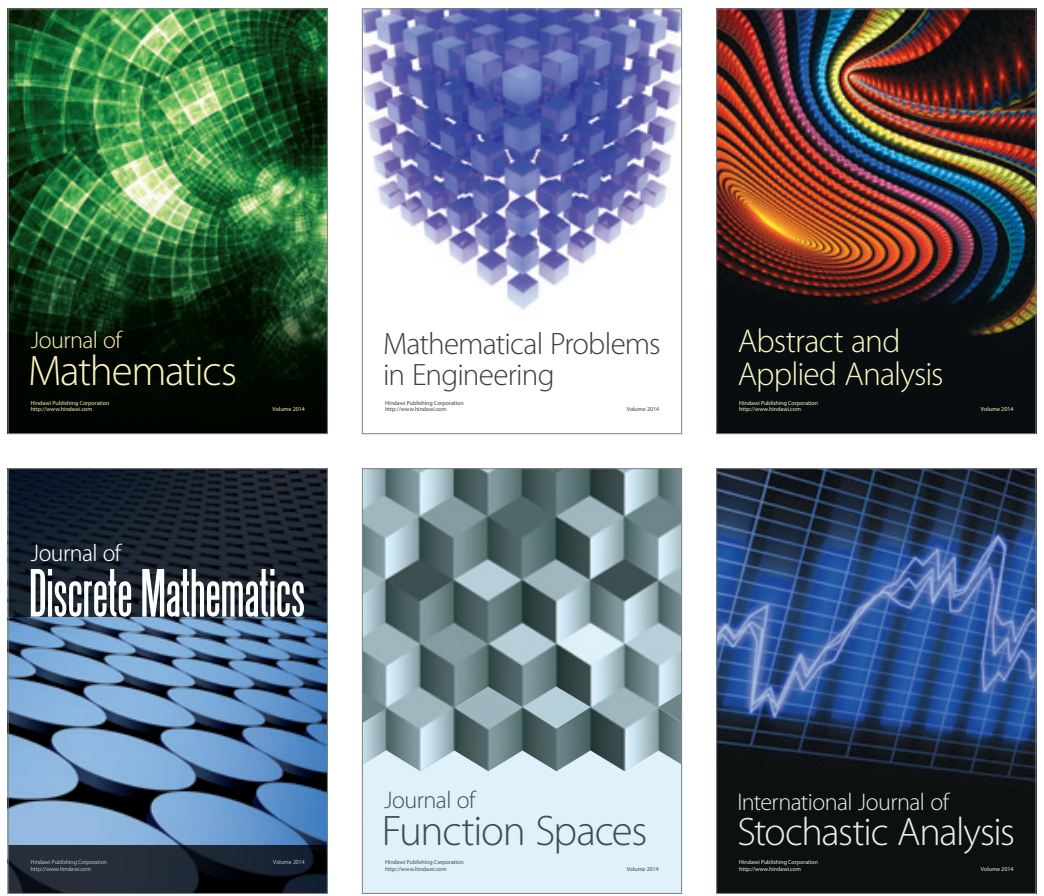

Journal of

Function Spaces

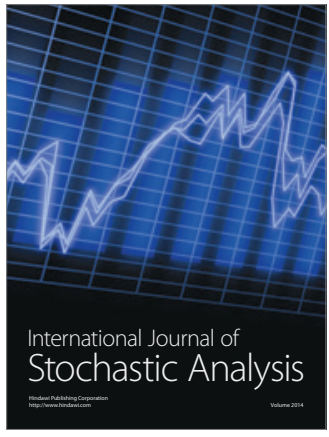

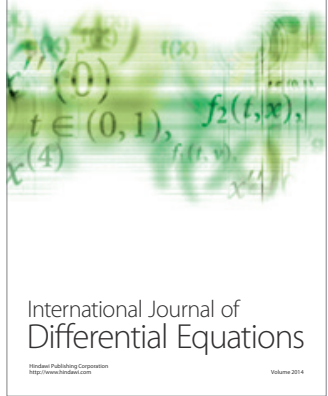
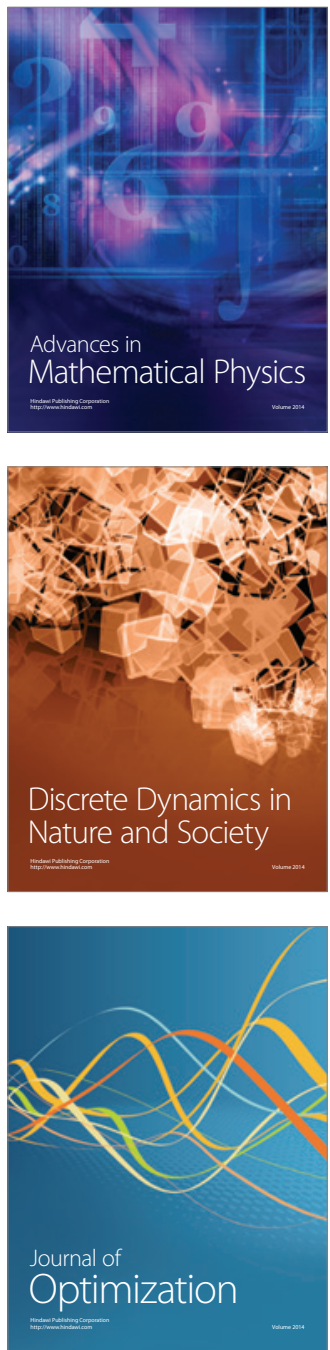